\title{
Quench protection analysis integrated in the design of dipoles for the Future Circular Collider
}

\author{
Tiina Salmi, ${ }^{1, *}$ Antti Stenvall, ${ }^{1}$ Marco Prioli, ${ }^{2}$ Janne Ruuskanen, ${ }^{1}$ Arjan Verweij, ${ }^{2}$ \\ Bernhard Auchmann, ${ }^{2}$ Davide Tommasini, ${ }^{2}$ Daniel Schoerling, ${ }^{2}$ Clement Lorin, ${ }^{3}$ \\ Fernando Toral, ${ }^{4}$ Maria Durante, ${ }^{3}$ Stefania Farinon, ${ }^{5}$ Vittorio Marinozzi, ${ }^{6}$ \\ Pasquale Fabbricatore, ${ }^{5}$ Massimo Sorbi, ${ }^{6}$ and Javier Munilla ${ }^{4}$ \\ ${ }^{1}$ Tampere University of Technology, Department of Electrical Engineering, \\ PL 52, 33101 Tampere, Finland \\ ${ }^{2}$ CERN, CH-1211 Geneva, 23, Switzerland \\ ${ }^{3}$ CEA Saclay, 91400 Saclay, France \\ ${ }^{4}$ CIEMAT, 28040 Madrid, Spain \\ ${ }^{5}$ INFN, 16146 Genoa, Italy \\ ${ }^{6}$ University of Milano and INFN-LASA, 20090 Milan, Italy \\ (Received 10 July 2016; published 27 March 2017)
}

\begin{abstract}
The EuroCirCol collaboration is designing a $16 \mathrm{~T} \mathrm{Nb}_{3} \mathrm{Sn}$ dipole that can be used as the main bending magnet in a $100 \mathrm{~km}$ long $100 \mathrm{TeV}$ hadron-hadron collider. For economic reasons, the magnets need to be as compact as possible, requiring optimization of the cable cross section in different magnetic field regions. This leads to very high stored energy density and poses serious challenges for the magnet protection in case of a quench, i.e., sudden loss of superconductivity in the winding. The magnet design therefore must account for the limitations set by quench protection from the earliest stages of the design. In this paper we describe how the aspect of quench protection has been accounted for in the process of developing different options for the $16 \mathrm{~T}$ dipole designs. We discuss the assumed safe values for hot spot temperatures and voltages, and the efficiency of the protection system. We describe the developed tools for the quench analysis, and how their usage in the magnet design will eventually ensure a secure magnet operation.
\end{abstract}

DOI: 10.1103/PhysRevAccelBeams.20.032401

\section{INTRODUCTION}

Superconducting dipole magnets with high magnetic field are used in synchrotron accelerators to keep the high-energy particles inside the ring-shaped beam pipe. Within the Future Circular Collider design study [1], the European Commission funded European Circular Collider study (EuroCirCol) [2] aims at designing a $16 \mathrm{~T} \mathrm{Nb}_{3} \mathrm{Sn}$ dipole magnet to guide two beams of $50 \mathrm{TeV}$ protons in a $100 \mathrm{~km}$ ring [3]. The dipole magnet conductor drives the overall cost of the accelerator [4]. Therefore, the magnet design aims at minimizing the amount of superconducting cable. As the magnets get more compact in size, the stored energy density increases and therefore also the temperature gradients and peak temperature during a quench. Quench protection is therefore an important aspect of the magnet design, giving a lower limit to the minimum conductor amount, and it

*tiina.salmi@tut.fi

Published by the American Physical Society under the terms of the Creative Commons Attribution 4.0 International license. Further distribution of this work must maintain attribution to the author(s) and the published article's title, journal citation, and DOI. must be considered from the very beginning. In particular, care must be taken that the peak temperature, temperature gradients and the voltages in the coil stay within safe limits. This paper discusses how the magnet protection was integrated in the first phase of designing the EuroCirCol dipole.

Three coil configurations are currently explored in the EuroCirCol study: $\operatorname{Cos} \theta$, block and common coil (CC) [5]. The quench protectability was quantified in a similar manner to all design options. Furthermore, the quench protection analysis was centralized and the same tools and analysis were applied to all of them. Quench simulation tools providing fast feedback were developed for the purpose of guiding the magnet design. The designed magnet underwent a protection study with certain assumed protection system efficiency and the resulting temperatures and voltages were analyzed and compared to the assumed safe values. In the definition of the safe values and obtainable protection efficiency we profited from the recent research for the $\mathrm{Nb}_{3} \mathrm{Sn}$ magnets developed for the high luminosity LHC (HL-LHC) [6]. In this paper these assumptions are discussed in Secs. II and III, the developed tools in Sec. IV, and the results for the designed magnets in Secs. V and VI. 


\section{MAXIMUM TEMPERATURES AND VOLTAGES DURING QUENCH}

\section{A. Maximum temperatures}

The maximum allowed temperature during a quench in $\mathrm{Nb}_{3}$ Sn magnets has been discussed for over a decade, since the high-field $\mathrm{Nb}_{3} \mathrm{Sn}$ magnet development began [7-9]. Conservative views highlight the $\mathrm{Nb}_{3} \mathrm{Sn}$ strain sensitivity and the lifetime effect of repeated thermal stresses. Those suggest limiting the temperature to 100-200 K [9]. However, the high temperature quench experiments on R\&D magnets have shown no impact on critical current for peak temperatures under $380 \mathrm{~K}$, and no significant permanent degradation under $400 \mathrm{~K}$, even after repeated quenching $[6,8]$. The temperature range at which effects were observed was associated with the glass transition temperature of the used epoxy (CTD-101), which is near $380 \mathrm{~K}$. Taking this as the limit, and adding a sufficient margin, a maximum temperature of $350 \mathrm{~K}$ was chosen for the HL-LHC magnets [8]. In this design study we adopted the same limit. It is important to note that the experimental temperatures were not directly measured but deduced from the measured current decay curves assuming adiabatic conditions. Therefore, the quench analysis should rely on a similar modeling approach. The stress distribution is of course magnet and cable specific, and detailed studies are foreseen. Indeed, the thermal stresses due to temperature gradients are still a largely unexplored topic. In this preliminary analysis we aim to quantify what kind of temperature gradients can be expected but we will not state which gradient can be tolerated.

\section{B. Maximum voltages}

The maximum voltages inside the coils are limited by the cable insulation dielectric strength as well as the coil ground insulation. If quench heaters are used, they are likely to be the grounded elements with the shortest distance to the cables. In EuroCirCol a $150 \mu \mathrm{m}$ glass cable insulation is taken as baseline [5], which is the same as in the HL-LHC quadrupoles. It is assumed that the coil insulation scheme will be able to withstand $2 \mathrm{kV}$ voltages (with sufficient margin). The maximum magnet voltage during a quench considered in this study is $1 \mathrm{kV}$, given that, when it is summed with the accumulated voltage of a magnet string, the $2 \mathrm{kV}$ threshold must not be exceeded. However, this study does not cover magnet strings. In HL-LHC magnets the limit for maximum voltages during quench has been considered to be $1 \mathrm{kV}$.

\section{EFFICIENCY OF THE PROTECTION SYSTEM}

The quench protection of the high-field magnets will require an active approach. The first step is to detect the quench, and then as quickly as possible discharge its current. The current discharge requires an increase of the circuit resistance to allow conversion of the stored magnetic energy into heat through Joule heating. An external dump resistor will not be able to extract a significant fraction of the energy because it would lead to a too large terminal voltage. The resistance driving the current decay must therefore be developed in the coil itself and the entire stored energy must be absorbed by the windings. To reduce thermal gradients, peak temperature and voltage the protection system must aim at bringing the entire winding to the normal state as fast and evenly as possible. This is the same approach as in the LHC and HL-LHC $\mathrm{Nb}_{3} \mathrm{Sn}$ magnets.

\section{A. Quench delays}

The efficiency of the protection system is characterized by the delay times associated with initiating quenches in the different coil regions. In this paper we use the term quench delay to refer to the sum of the detection delay, related to detecting the initial quench and activating the protection system, and the heater delay, or CLIQ delay, related to the protection system (namely, the heaters, or CLIQ) provoking a quench after its activation. The description of both protection system types is given below.

If the quench delay is uniform in all coil turns, it means that after that time the entire winding is at once brought to the normal state. Estimating a uniform quench delay which represents the protection system efficiency is useful because it can be used to define the minimum time margin [10] that the magnet must have in order to keep the peak temperature below $350 \mathrm{~K}$.

\section{B. Quench detection}

The quench detection is assumed to be based on the detection of the resistive voltage associated with the initial normal state transition. Based on the experience of the noise level and electronic system delay times in the LHC, $20 \mathrm{~ms}$ was assumed to be the delay time to the activation of the quench protection system at nominal operation current.

\section{Quench protection methods}

The considered quench protection methods are resistive protection heaters, the coupling loss induced quench protection system (CLIQ) [11] or their combination. Protection heaters are a well-established technology that is used also in the LHC. They are resistive strip heaters that are positioned on the coil surface, and upon quench detection they are powered with a capacitor discharge [12]. The quench delay then depends on the heat diffusion through the insulation layers to the cables. CLIQ is a novel method that has shown promising results on the R\&D models of the HL-LHC magnets and it is foreseen to be applied for the HL-LHC inner triplet together with heaters [13]. CLIQ is based on discharging a capacitor into the coil causing oscillations of the magnet current which induce 
interfilament and interstrand coupling losses that spread the quench zone throughout the superconducting coil.

In order to estimate an obtainable quench delay to guide the first rounds of the magnet design, an analysis was performed considering protection heaters which cover each turn in the magnet. The heater technology was assumed similar to the HL-LHC magnets: $25 \mu \mathrm{m}$ stainless steel strips isolated from the coil with a layer of polyimide [14]. The polyimide thickness was $50 \mu \mathrm{m}$ in the HL-LHC inner triplet but here we considered up to 2 times larger thicknesses to account for the expected higher voltages.

\section{Heater delays}

To estimate the influence of heater delay on quench delay, we simulated the typical delays in the designed magnets. The expected heater delays were estimated using simulations with code for heater delay analysis (CoHDA) [14]. The simulation was performed by computing the power generation in the heater and the heat diffusion to the cable. The heater delay was defined as the time to increase the cable temperature above the current sharing temperature. This tool has been used for analysis and design of the heaters for HL-LHC magnets and the simulated delays have been in good agreement with measurements at high magnet current [15].

Figure 1 shows the simulated delays at nominal current as a function of magnetic field. The simulation was done for one of the first $\cos \theta$ and block-type coil designs that were produced in EuroCirCol. Cable 1 and cable 2 refer respectively to the different cables used in the high-field and low-field region of the coils. Note that the field range in Fig. 1 is wider than the field ranges that the cables actually see in the magnet at nominal current.

Two cases for the heater power and insulation were considered. Case A has $150 \mathrm{~W} / \mathrm{cm}^{2}$ peak power and $50 \mu \mathrm{m}$ insulation thickness, which is the same as in HL-LHC and is considered optimistic. In case B the peak power is lower, $50 \mathrm{~W} / \mathrm{cm}^{2}$, to account for longer heater strips, and the insulation thickness is $100 \mu \mathrm{m}$, to account for the higher quench voltages. For case A the average

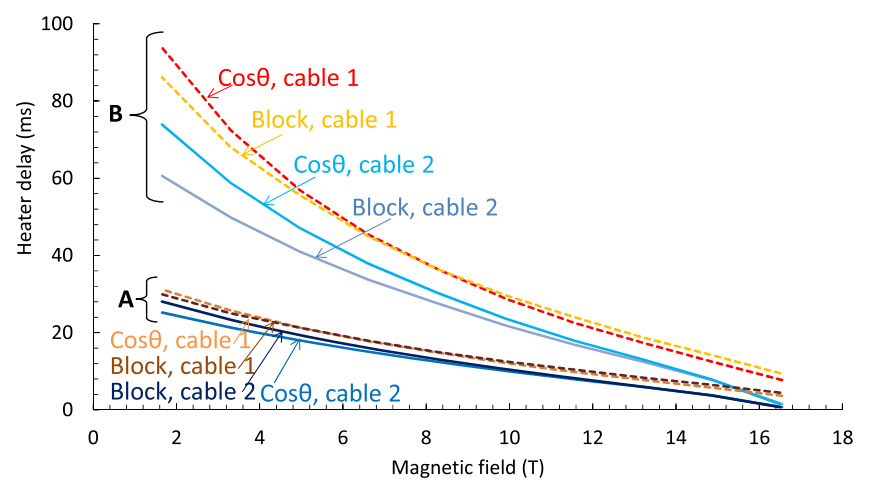

FIG. 1. Estimation of heater delays at nominal current. delays in the coil blocks were 6-22 $\mathrm{ms}$ and for case B 12-51 ms. In the coil turns with low field (always in cable 2), the delays can be as high as in Fig. 1, but the average delays will be significantly faster.

As a representative delay time of the protection system efficiency we chose $20 \mathrm{~ms}$, which represents the average achieved with simulations. This led us to set as a design criterion that the magnets must have minimum $40 \mathrm{~ms}$ time margin (including $20 \mathrm{~ms}$ for detection and activation delay). To keep the analysis simple in the conceptual design phase, the quench delay of $40 \mathrm{~ms}$ was used in the initial fast-feedback simulations. Some coil designs were also simulated with cable-specific quench delay, where the heater delay was computed with CoHDA to consider the reliability of the fast-feedback approach. First simulations with CLIQ later showed the adequateness of $40 \mathrm{~ms}$ quench delays in fast-feedback simulations in the case of CLIQ-based protection too.

\section{ANALYSIS METHODS AND TOOLS}

Two new tools were developed for the analysis of quench temperatures and voltages: QuenchProtection spreadsheet (QP-sheet) and code for current decay calculation based on known protection efficiency (Coodi) [16]. QP-sheet is used for fast feedback of the worst case hot spot temperature. It is implemented as a Microsoft Excel spreadsheet and was used by the magnet designers. If the temperature in the magnet was not within the limits with the $40 \mathrm{~ms}$ quench delay, it provided fast feedback how, for example, modifying the $\mathrm{Cu}$ content in the cable impacts the temperature.

Coodi is a Fortran 90 code and it enables a more detailed analysis of the temperature distribution, and failure scenarios, such as asymmetric heater failures, as well as voltages. Both use a very similar method for the temperature calculation. In principle coodi allows for longitudinal quench propagation (like between heating stations) but in this analysis it was assumed that the coil turns quench completely after the quench delay in each turn. Both tools rely on ROXIE [17] calculation of the magnetic field distribution and of the differential inductance. The quench detection times and heater delays were an input.

\section{A. Temperature calculation}

The coil temperatures after a quench were calculated adiabatically. This means that the Joule heat generated in each turn was completely absorbed by the cable cross section. The heat capacity included the strands, the cable insulation and the voids between strands (which was modeled as insulation in the impregnated magnets). The temperature increase $\Delta T$ at each time step $\Delta t$ was obtained from

$$
\Delta T=I_{\mathrm{mag}}^{2} \Delta t \frac{\rho_{\mathrm{Cu}}}{A_{\text {Cable }}^{2} f_{\mathrm{Cu}} C_{v}},
$$


where $I_{\mathrm{mag}}$ is the magnet current at that time instant. The current is assumed to flow in the copper matrix after the cable quenches. The term $\rho_{\mathrm{Cu}}$ is the copper resistivity, $A_{\text {cable }}$ is the cross-sectional area of the insulated cable, $f_{\mathrm{Cu}}$ is the cable volumetric copper fraction and $C_{v}$ is the volumetric specific heat of the cable computed as the weighted average of the cable material components. The resistivity and specific heat depend on local temperature and magnetic field, and are based on NIST data [18]. This equation is solved for each coil turn in Coodi, and in the spreadsheet for coil blocks that are considered at uniform temperature and field.

\section{B. Coil resistance and current decay}

When the temperature and magnetic field distribution are known, the resistance of each turn and thus the resistance of the magnet can be computed. The current decay between consecutive time steps can then be computed as

$$
I_{\mathrm{mag}}(t+\Delta t)=I_{\mathrm{mag}}(t) e^{-\Delta t R(t) / L(I)},
$$

where $R(t)$ is the magnet total resistance (if an external dump resistor was used it would be added to this term), and $L(I)$ is the magnet inductance accounting for the iron saturation at different currents. The inductance and resistance are assumed constant during the time step. The magnetic field was assumed to scale linearly with the current. Until the detection time the current was assumed to stay at its initial value. Note that the quench delay for each turn is an input, and the quench propagation or heat diffusion was not calculated.

\section{Voltage calculation}

The voltage of each coil turn consists of a resistive and an inductive component. To be able to study different fault scenarios in the future, the magnet was discretized to the half-turn level. The resistive part can be computed using the Ohm's law, knowing the resistance of each turn (using the average current during the time step). The inductive component requires information of the mutual inductances between the coil turns that were connected in series. This computation is done beforehand with a software introduced in [19]. It produces the effective inductance $L_{\text {eff }}$ for each turn, which includes the self-inductance of the turn and the contribution of the mutual inductances from the other turns. The distribution of the inductances was computed without the iron contribution, and it was assumed that their relative portion of the magnet total inductance stays the same even at low current where the absence of iron saturation increases substantially the differential inductance. After all, the largest voltages occur before the low-current regime is reached. The voltage at each half turn, $i$, was computed as

$$
V_{i}=V_{\mathrm{res}, i}+V_{\mathrm{ind}, i}=R_{i} I_{\mathrm{mag}}+L_{\mathrm{eff}, i} \frac{\Delta I_{\mathrm{mag}}}{\Delta t} .
$$

The potential to ground at each turn was obtained by summing the turns' total voltages according to their electrical order, starting from the negative current lead. The internal voltages between the turns are subsequently obtained from the difference in potential between them.

\section{ANALYSIS OF THE MAGNETS}

Throughout the magnet design the QP-sheet was used by the magnet designers to ensure the magnets had the sufficient amount of copper to limit the peak temperature. The detailed temperature distributions and voltages were subsequently analyzed then for all three magnet options using Coodi. The magnet protection analysis was performed at $105 \%$ of nominal current $\left(I_{\text {nom }}\right)$, to allow their testing above nominal current. This section summarizes the magnet parameters and presents results of the analysis.

\section{A. Magnet parameters}

The three design options, $\operatorname{Cos} \theta$ [20], block [21] and CC [22] are all graded multilayer coils, see Fig. 2. The grading in the $\cos \theta$ design is done so that the two higher field layers (inner layers seen from the bore) are wound using a highfield (HF) cable - called cable 1. It has a larger amount of superconductor than the low-field (LF) cable (cable 2). This saves in the superconductor cost and helps to quench the (a)
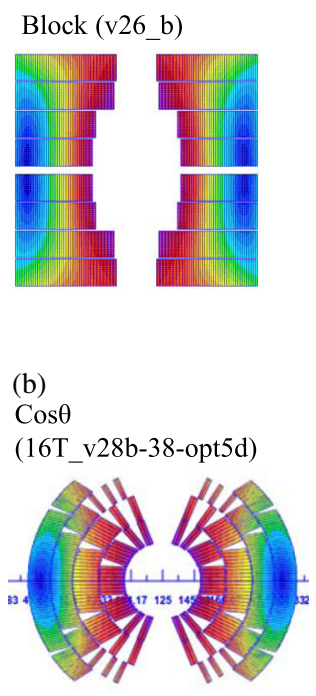

(c)

CommonCoil (v1h_intragrad_t2)
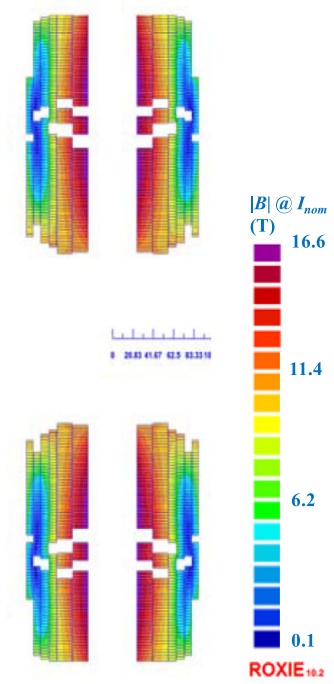

FIG. 2. Cross sections and magnetic field distribution of one aperture of (a) block and (b) $\operatorname{Cos} \theta$ design. For the $\mathrm{CC}$ design, (c), both apertures are shown. The coils are not to scale relative to each other. The color map gives an approximate value for the different field regions. In parentheses we show the coil design version identifiers for relating them to subsequent literature. 
TABLE I. Cable parameters for quench simulation of the magnets (insulated area and material fractions).

\begin{tabular}{lcccc}
\hline \hline Design, cable & $A_{\text {cable }}\left(\mathrm{mm}^{2}\right)$ & $f_{\mathrm{Cu}}$ & $f_{\text {Nb3Sn }}$ & $f_{G 10}$ \\
\hline Block, cable 1 & 32.5 & 0.36 & 0.36 & 0.27 \\
Block, cable 2 & 21.9 & 0.34 & 0.34 & 0.33 \\
Cos $\theta$, cable 1 & 38.0 & 0.36 & 0.36 & 0.28 \\
Cos $\theta$, cable 2 & 22.4 & 0.45 & 0.22 & 0.32 \\
CC, cable 1 & 33.9 & 0.36 & 0.36 & 0.29 \\
CC, cable 2 & 23.2 & 0.43 & 0.25 & 0.32 \\
CC, cable 3 & 19.0 & 0.51 & 0.15 & 0.34 \\
CC, cable 4 & 19.0 & 0.53 & 0.13 & 0.34 \\
\hline \hline
\end{tabular}

TABLE II. Magnet nominal operation current and differential inductance for two apertures. The average stored energy density $E$ is computed considering the volume of the insulated cable.

\begin{tabular}{lrccc}
\hline \hline & $\begin{array}{r}I_{\text {nom }} \\
(\mathrm{kA})\end{array}$ & $\begin{array}{c}L \\
(\mathrm{mH} / \mathrm{m})\end{array}$ & $\begin{array}{c}E \\
\left(\mathrm{~J} / \mathrm{mm}^{3}\right)\end{array}$ & $\begin{array}{c}J_{\mathrm{Cu}}, \text { cable1-2-3-4 } \\
\left(\mathrm{kA} / \mathrm{mm}^{2}\right)\end{array}$ \\
\hline Block & 8.4 & $2 \times 42.5$ & 0.11 & $0.7-1.1$ \\
$\operatorname{Cos} \theta$ & 10.3 & $2 \times 26.0$ & 0.11 & $0.87-1.0$ \\
$\mathrm{CC}$ & 9.0 & 110.0 & 0.12 & $0.7-0.9-0.9-0.9$ \\
\hline \hline
\end{tabular}

coil cross section more uniformly. In the $\mathrm{CC}$ coil four different cables are used, cable 1 for the innermost layer, cable 2 for the second layer, cable 3 for the next two layers and cable 4 for the two outermost layers. In the block design the grading is done within the layers. The innermost turns of each layer are wound using the thicker HF cable (cable 1) and the outer turns using cable 2. Tables I and II detail the cable and operation parameters. The magnets are operated at $4.5 \mathrm{~K}$.

\section{B. Hotspot temperature vs quench delay}

The hot spot temperature was analyzed at $105 \%$ of nominal operation current, as a function of uniform quench delay. Figure 3 shows the worst case hot spot temperature, which occurs when the initial normal zone is located in the low-field cable with the highest field. This calculation is done using the QP-sheet.

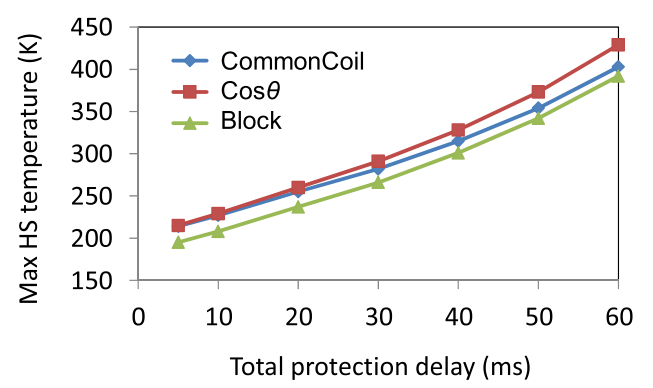

FIG. 3. Maximum peak temperature vs protection quench delay.
The temperatures increase by $30-50 \mathrm{~K}$ for each additional $10 \mathrm{~ms}$ in quench delay. Even if the total delay would only be $10 \mathrm{~ms}$, then the peak temperature would still be above $200 \mathrm{~K}$. The behavior of all the magnets is similar. With $40 \mathrm{~ms}$ quench delay all the temperatures are below $350 \mathrm{~K}$, i.e., the time margins of each design are within the requirements.

\section{Distributed heater delays}

The distribution of the temperatures and voltages in the coil cross section were further analyzed using coodi to confirm the relevance of the fast-feedback analysis with the state-of-the-art designs. In addition to the uniform $40 \mathrm{~ms}$ quench delay, a case with a quench delay distribution based on protection heaters was considered. The heaters provided the same heat input to each cable from the cable's narrow sides. Therefore, the quench delay at each cable was approximately proportional to the margin to quench. The heat input was defined assuming a heater peak power of $100 \mathrm{~W} / \mathrm{cm}^{2}$ and a polyimide insulation to coil of $75 \mu \mathrm{m}$ thickness. The delays were simulated for each magnet using CoHDA.

Figures 4-6 show the simulated heater delays and the total quench delays with $20 \mathrm{~ms}$ detection time in each
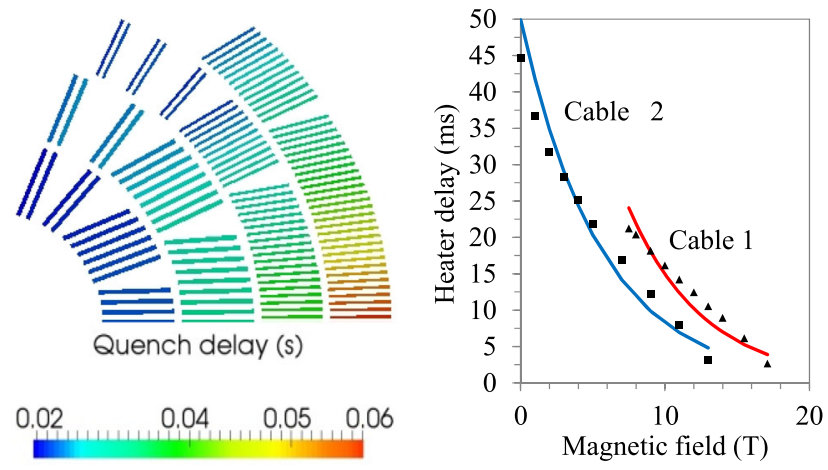

FIG. 4. Quench delay and heater delay at $105 \%$ of $I_{\text {nom }}$ in the $\operatorname{Cos} \theta$ design.
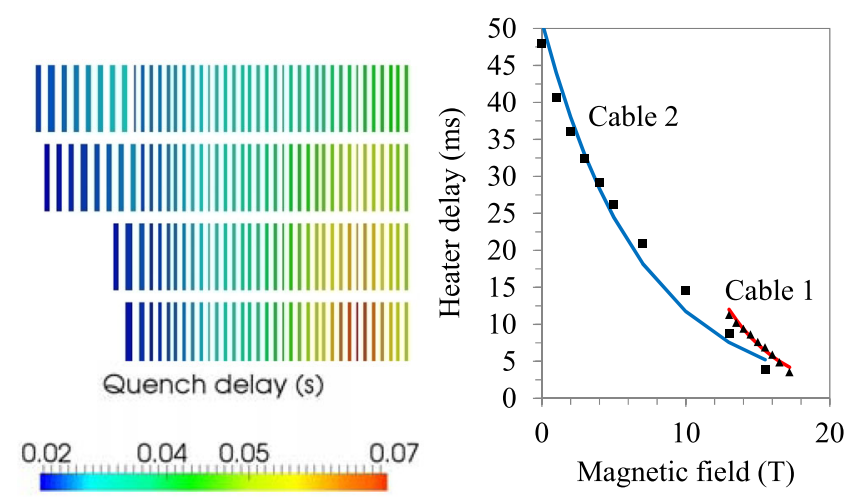

FIG. 5. Quench delay and heater delay at $105 \%$ of $I_{\text {nom }}$ in the block design. 

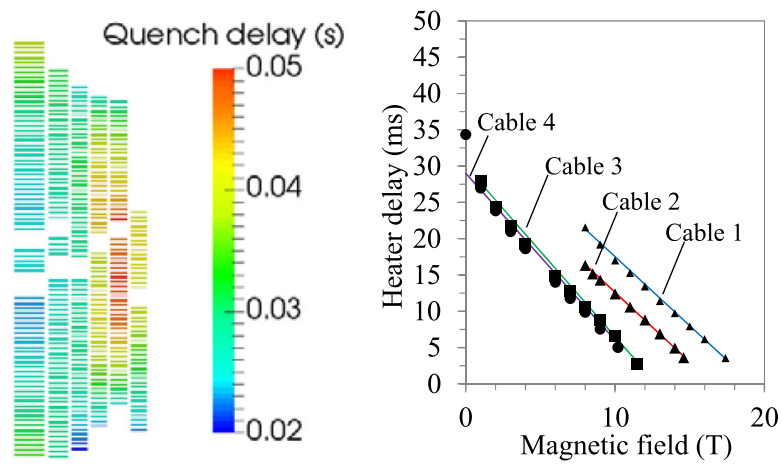

FIG. 6. Quench delay and heater delay at $105 \%$ of $I_{\text {nom }}$ in the common-coil design.

magnet. The quench delay depends strongly on the field and cable type. In all cases the shortest heater delays are 3-4 ms, and are located in the high-field region. The average heater delay in the coil cross section is $14 \mathrm{~ms}$ in the $\operatorname{Cos} \theta, 18 \mathrm{~ms}$ in the block, and $14 \mathrm{~ms}$ in the CC design.

\section{Simulated temperatures}

The results of Coodi simulations showing the final temperatures in the magnets after a quench are shown in Figs. 7-9. The hot spot temperatures are not shown, but are listed in Table III. They were between 295 and $330 \mathrm{~K}$ in all cases.

Excluding the hot spot, the simulated temperatures were in the range from 70 to $200 \mathrm{~K}$ in the case of uniform $40 \mathrm{~ms}$ quench delay and from 70 to $220 \mathrm{~K}$ in the case of distributed heater delay. The lowest temperatures are always in the high-field cable which has a higher copper content, and the highest temperatures are in the low-field cable with the highest field and highest $J_{\mathrm{Cu}}$ during quench. The largest gradients are also between the high- and lowfield cables, and are about $100 \mathrm{~K}$. In the $\operatorname{Cos} \theta$ and $\mathrm{CC}$ designs the gradient is between cables in different layers, where the insulation thickness is larger, but in the block design the gradient is between laterally adjacent turns.

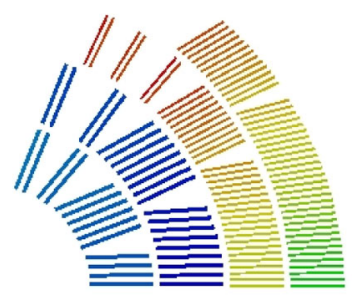

Temperature $(K)$

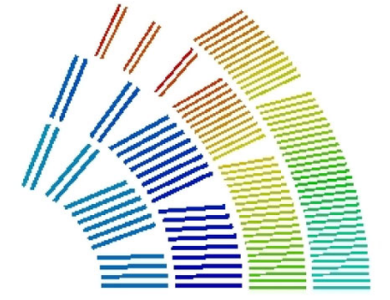

Temperature $(\mathrm{K})$

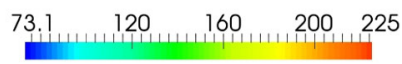

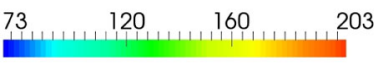

FIG. 7. Final temperature $(\mathrm{t}=600 \mathrm{~ms})$. Left: Uniform $40 \mathrm{~ms}$ delay; right: distributed heater delay.

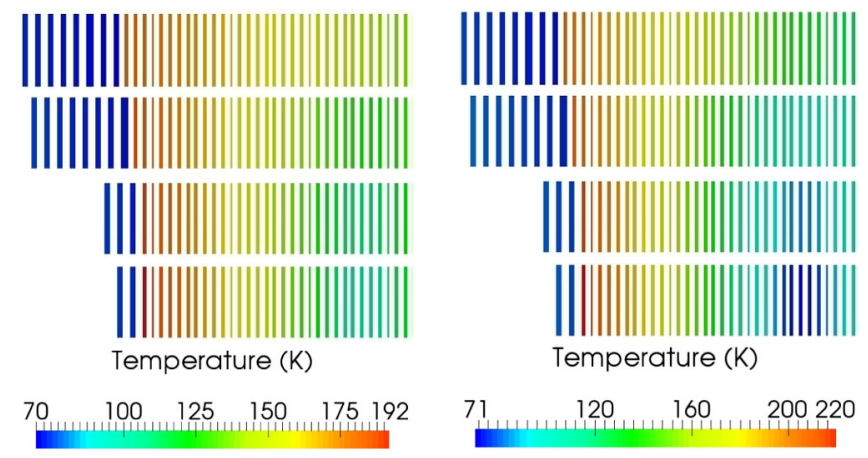

FIG. 8. Final temperature $(\mathrm{t}=550 \mathrm{~ms})$. Left: Uniform $40 \mathrm{~ms}$ delay; right: distributed heater delay.

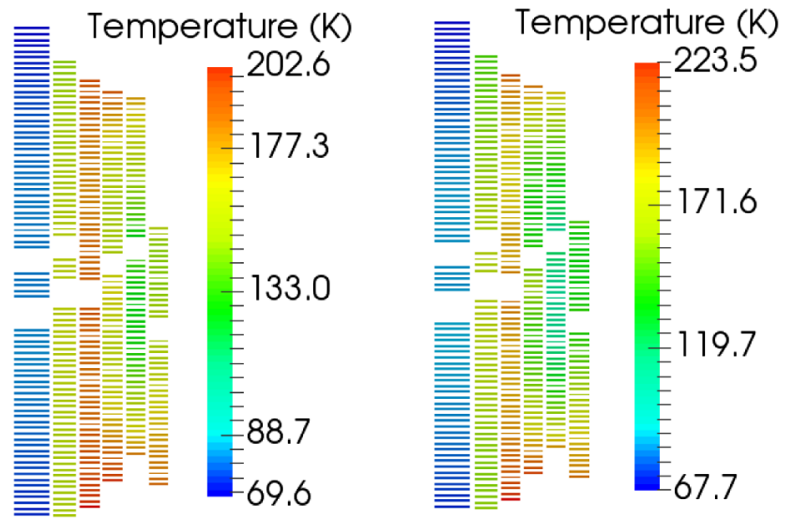

FIG. 9. Final temperature $(\mathrm{t}=600 \mathrm{~ms})$. Left: Uniform $40 \mathrm{~ms}$ delay; right: distributed heater delay.

The larger $\mathrm{Cu}$ content in high-field cable is partially because of the design limitation to use a $\mathrm{Cu}$-to-non- $\mathrm{Cu}$ ratio of minimum 1. Therefore, the larger amount of needed superconductor in the high-field cable required also a larger area of copper. Lowering the $\mathrm{Cu}$-to-non-Cu ratio could lead to more uniform temperature distribution.

It is worth noting that the performed analysis was conservative because the heat diffusion was neglected. Further analysis will be performed later by combining mechanical and thermal stresses.

\section{E. Calculated voltages}

If the resistive and inductive voltages were distributed equally in all turns, then the potential to ground would be

TABLE III. Summary of simulated peak temperatures and voltages after a simulation with uniform $40 \mathrm{~ms}$ quench delay.

\begin{tabular}{lcccc}
\hline \hline Magnet & $T_{\max }(\mathrm{K})$ & $V_{\text {gnd }}(\mathrm{kV})$ & $V_{\text {turn-to-turn }}(\mathrm{V})$ & $V_{\text {layer-to-layer }}(\mathrm{kV})$ \\
\hline Block & 310 & $-1.2 \ldots 1.2$ & 80 & 1.1 \\
$\operatorname{Cos} \theta$ & 330 & $-1.4 \ldots 0.4$ & 100 & 1.8 \\
$\mathrm{CC}$ & 320 & $-2.3 \ldots 1.4$ & 80 & 3.3 \\
\hline \hline
\end{tabular}




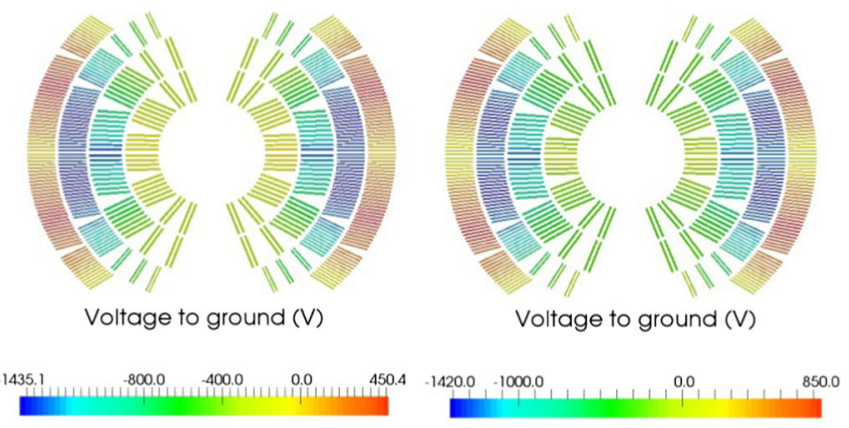

FIG. 10. Voltage to ground in the $\operatorname{Cos} \theta$ design at $\mathrm{t}=180 \mathrm{~ms}$. Left: Uniform $40 \mathrm{~ms}$ delay; right: distributed heater delay.

zero at all locations. Resistive voltages vary of course always in a magnet with different cables, magnetic field regions, and quenching times. Furthermore, the inductive voltage cannot be uniformly distributed. The inductance at coil outer turns, where the field is lower, is typically 2-3 times larger than in the inner turns, with higher field. This is problematic for the voltages because the lower field regions will have less heating due to the lower resistivity and also tend to have longer heater delays due to larger temperature margin. However, in these graded coils more heat is generated in the low field regions due to lower $\mathrm{Cu}$ area in the cable.

Figures 10, 12, and 14 show the voltage distributions near the time instant when the potential to ground reached its highest value.

\section{1. $\operatorname{Cos} \theta$ design}

In the $\operatorname{Cos} \theta$ design the peak voltage to ground reached $1.4 \mathrm{kV}$ around $180 \mathrm{~ms}$ after the initial quench. The location is in the inner low-field cable layer (third layer from the bore).

At the same time instant, the temperature distribution is similar to the one in Fig. 7, and ranges from 65 to $151 \mathrm{~K}$ with the uniform quench delay, and from 65 to $172 \mathrm{~K}$ with distributed delays. Figure 11 shows the distribution of the resistive voltage in each turn which is similar to the temperature distribution, as expected. The resistive voltage in the $14 \mathrm{~m}$ long turns is below $20 \mathrm{~V}$ in cable 1 , while reaching 60 to $80 \mathrm{~V}$ in the high-field locations of cable 2 . The slightly higher peak voltage in the case of the distributed heater delay can be explained by faster heater delay (less than $20 \mathrm{~ms}$ ) in the high-field area of cable 2.

The total inductive voltage is defined by the magnet total inductance and the current decay rate. In general, larger inductive voltages occur at the outer midplane turns of the magnet, since the area of the loops, and total flux through them, is larger. The inductive component in the turns varies between -10 and $-50 \mathrm{~V}$. The inductive voltage could be more effectively compensated by quenching the outer lowfield turns first so that the higher temperatures would lead to larger resistive voltages. However, this is clearly in
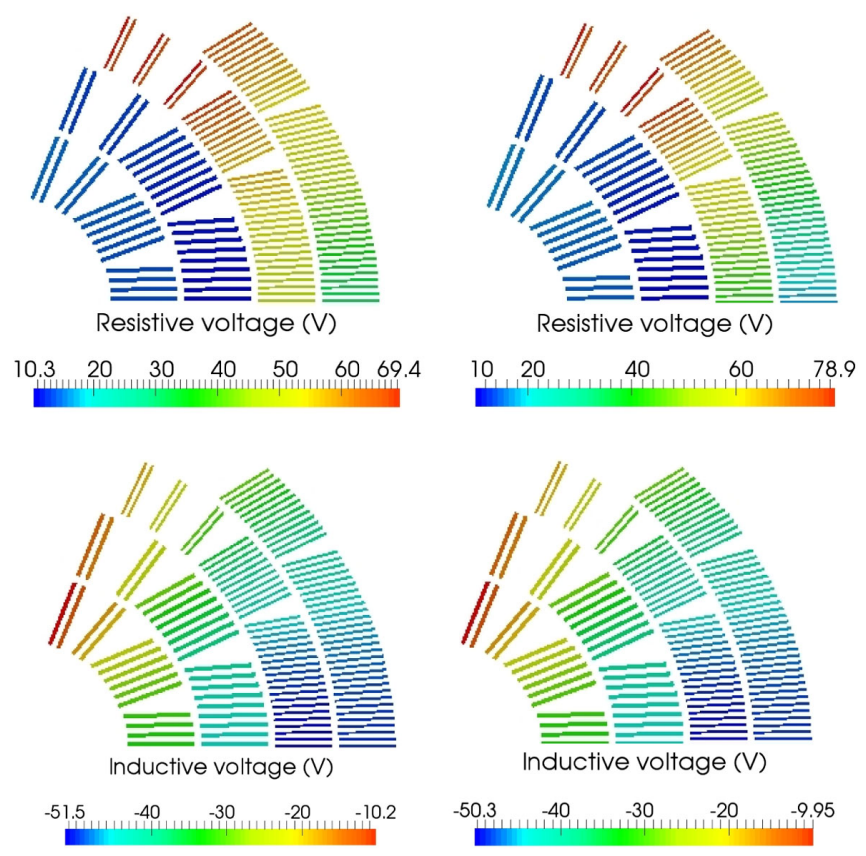

FIG. 11. Resistive and inductive voltages across the coil half turns at $\mathrm{t}=180 \mathrm{~ms}$. Left: Uniform $40 \mathrm{~ms}$ delay; right: distributed heater delay.

conflict with the need of a fast current decay, requiring to initiate and propagate the quench as fast as possible, which is easiest in the high-field region.

\section{Block design}

In the block design the peak voltage to ground occurs also inside the magnet (Fig. 12). Contrary to the $\operatorname{Cos} \theta$ and $\mathrm{CC}$ designs, in the block design the peak voltage is distributed evenly around $0 \mathrm{~V}$. This is because of the winding order. In the $\cos \theta$ coils the current lead enters both coils in the inner layer outermost turn. That leads to identical accumulation of voltages in both coils. In the block design however, the winding order is reversed in the upper and lower coil.

As expected, the distribution of the resistive voltage again follows closely the temperature distribution (Fig. 13).

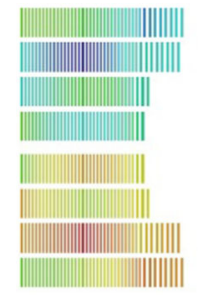

Voltage to ground $(V)$

$\begin{array}{llllll}-1173 & -800 & -400 & 0 & 400 & 800 \quad 1173\end{array}$

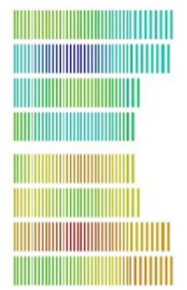

Voltage to ground $(V)$

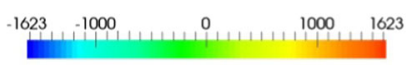

FIG. 12. Voltage to ground at $\mathrm{t}=160 \mathrm{~ms}$. Left: Uniform $40 \mathrm{~ms}$ delay; right: distributed heater delay. 

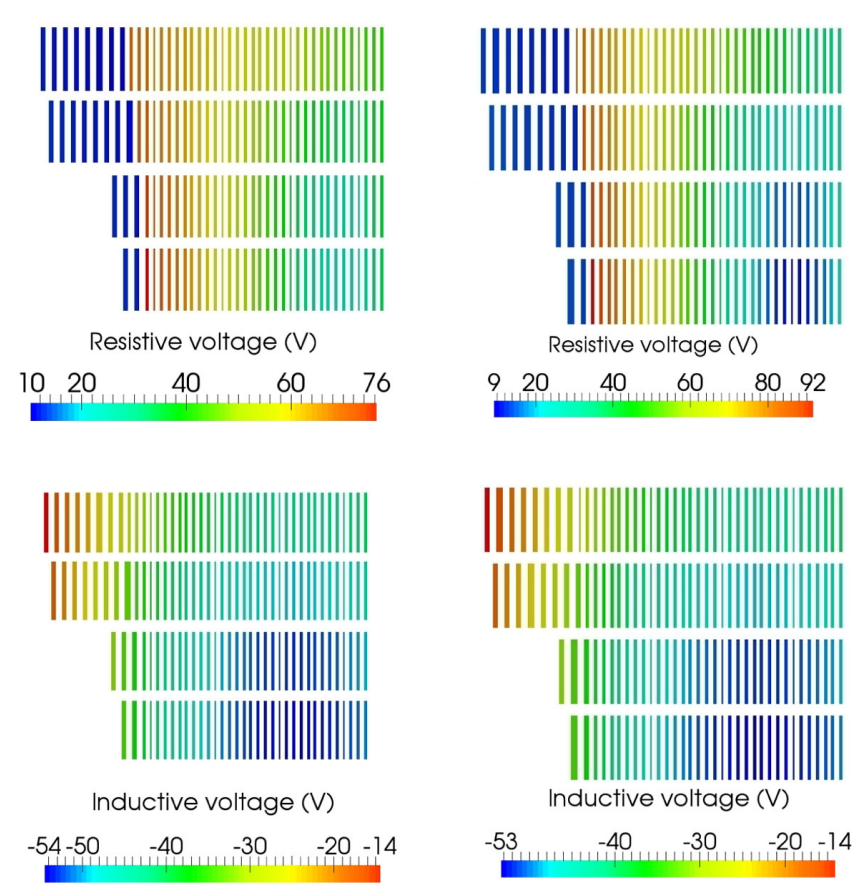

FIG. 13. Resistive and inductive voltages across the half turns at $\mathrm{t}=160 \mathrm{~ms}$. Left: Uniform $40 \mathrm{~ms}$ delay; right: distributed heater delay.

The resistive voltage in the HF cable is around $10-15 \mathrm{~V}$, but in the LF cable reaches $80-90 \mathrm{~V}$. This is again due to the smaller $\mathrm{Cu}$ section in the LF cable.

Also in the block design the largest inductive voltages occur near the midplane in the outer turns. At $160 \mathrm{~ms}$ after the initial quench the inductive there is $-53 \mathrm{~V}$, and in the inner pole turns it is $-14 \mathrm{~V}$. The temperatures at that time instant have a similar distribution as in Fig. 8. The temperatures range from 60 to $140 \mathrm{~K}$ with uniform delay, and from 50 to $160 \mathrm{~K}$ with distributed delay.

\section{Common coil}

In the $\mathrm{CC}$ design the potential difference to ground reaches $(-) 2.3 \mathrm{kV}$ in the case with $40 \mathrm{~ms}$ uniform quench delay, and $(+) 2.7 \mathrm{kV}$ in the case with distributed heater delays.
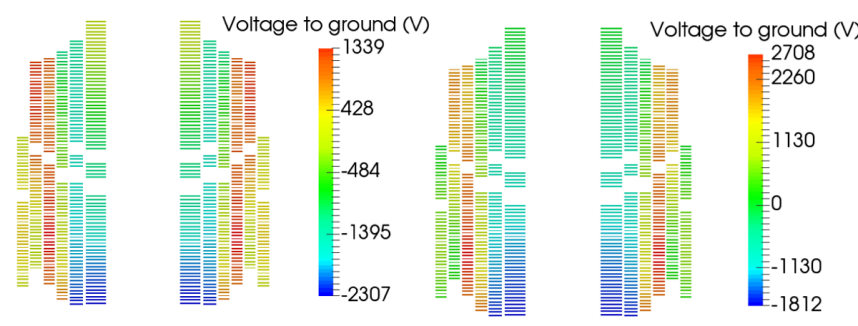

FIG. 14. Voltage to ground in the common coil. Left: Uniform $40 \mathrm{~ms}$ delay, $\mathrm{t}=200 \mathrm{~ms}$; right: distributed heater delays, $\mathrm{t}=160 \mathrm{~ms}$. Only upper halves of coils are shown (see Fig. 2).
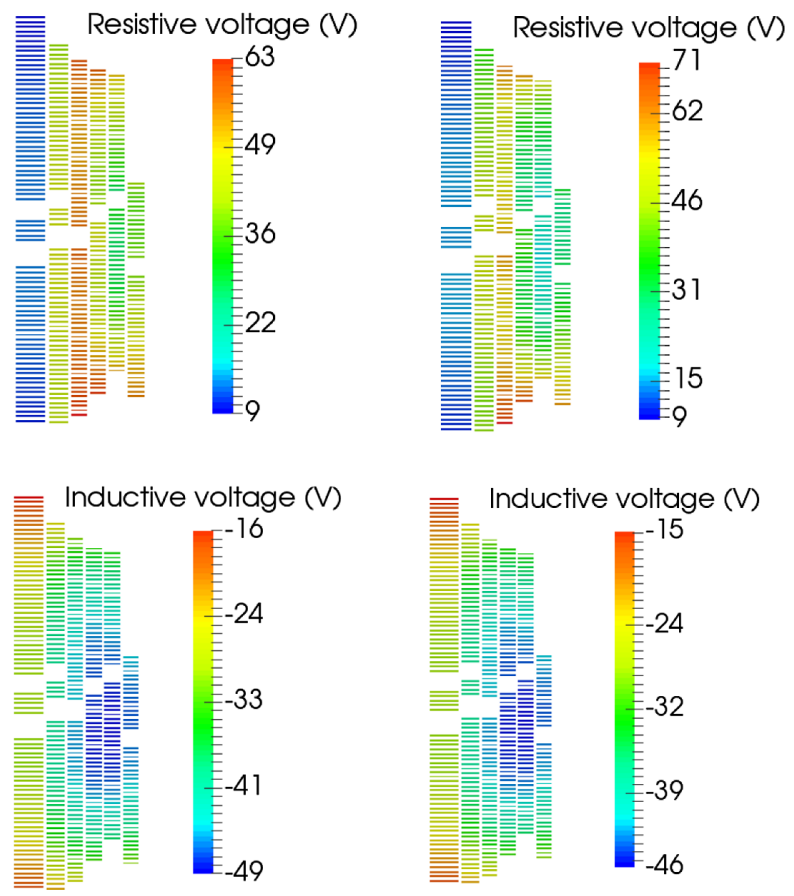

FIG. 15. Resistive and inductive voltages across the half turns. Left: Uniform $40 \mathrm{~ms}$ delay, $\mathrm{t}=200 \mathrm{~ms}$; right: distributed heater delays, $\mathrm{t}=160 \mathrm{~ms}$.

The temperatures in the CC design at the moment of the peak voltage are similar to those of the block and $\cos \theta$ designs. The lowest temperatures in the HF cable 1 are $60-70 \mathrm{~K}$, and reach $140 \mathrm{~K}$ in the LF cable.

Both the resistive and inductive voltages (Fig. 15) have a similar range as compared to the other two designs. The larger peak voltage to ground is probably because of large blocks (layers) where a large number of predominantly positive or negative turns are summed one after the other.

\section{Summary}

Tables III and IV summarize the peak temperatures, peak voltage to ground, and peak voltage between adjacent turns and layers.

The temperatures are well below the allowed limit. But the voltages are above the allowed limit even in these nominal cases. When failure cases will be introduced to the analysis, they are likely to increase significantly above the

TABLE IV. Summary of simulated peak temperatures and voltages after a simulation with quench delay based on simulated heater delays in each turn.

\begin{tabular}{lcccc}
\hline \hline Magnet & $T_{\max }(\mathrm{K})$ & $\left|V_{\text {gnd }}\right|(\mathrm{kV})$ & $V_{\text {turn-to-turn }}(\mathrm{V})$ & $V_{\text {layer-to-layer }}(\mathrm{kV})$ \\
\hline Block & 300 & 1.6 & 110 & 1.6 \\
Cos $\theta$ & 305 & 1.4 & 123 & 2.2 \\
CC & 293 & 2.7 & 93 & 4.1 \\
\hline \hline
\end{tabular}


$1 \mathrm{kV}$ limit. Therefore, several possibilities to reduce these voltages were studied in detail.

\section{CLIQ}

The suitability of the adopted quench protection assumptions was verified also in the case of a protection based on the CLIQ technology. It is a new technology for the protection of superconducting magnets that has been developed at CERN [11]. It relies on interfilament and interstrand coupling currents to spread quickly the normal zone after a quench. In this section we look at the performance of CLIQ when applied to the block design.

The CLIQ technology can be applied to all of the designed magnet options. The block-type dipole was chosen as the first modeling case to demonstrate the CLIQ compatibility with the chosen design criterion. The rest of the magnets will be covered at a later stage of the magnet design study, as well as the optimization of the CLIQ configuration.

\section{A. Connecting CLIQ to the magnet}

As shown in Fig. 16, the core component of CLIQ is the capacitor bank that generates an alternated transport current in the magnet and, consequently, a variable magnetic field in the coils, resulting in interfilament and interstrand coupling losses.

The block coil has been chosen as the benchmark design to verify if CLIQ technology can be considered for the protection of high-field high-energy superconducting magnets and to compare its performances with the ones of quench heaters discussed in the previous section.

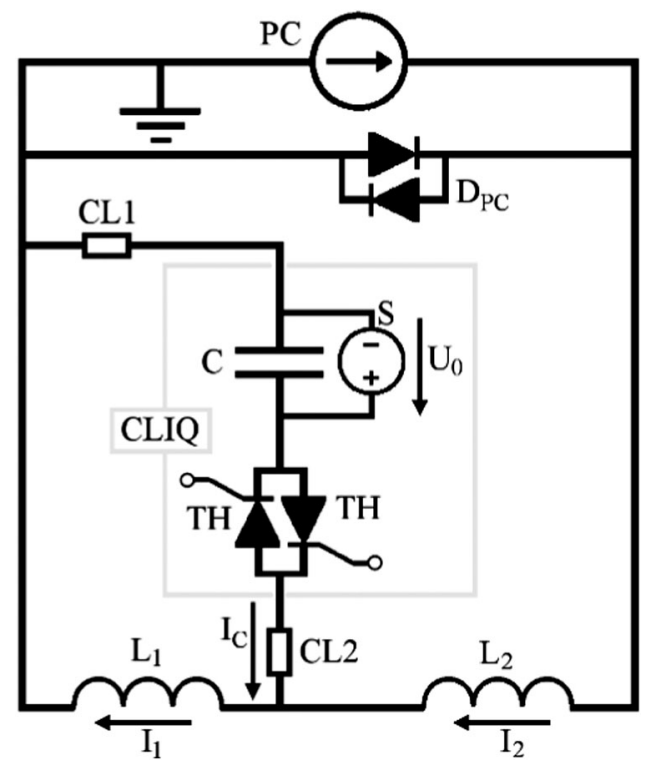

FIG. 16. Schematic of a CLIQ unit connected to a superconducting magnet. $\mathrm{L}_{1}$ and $\mathrm{L}_{2}$ represent two different electrical parts of the magnet.

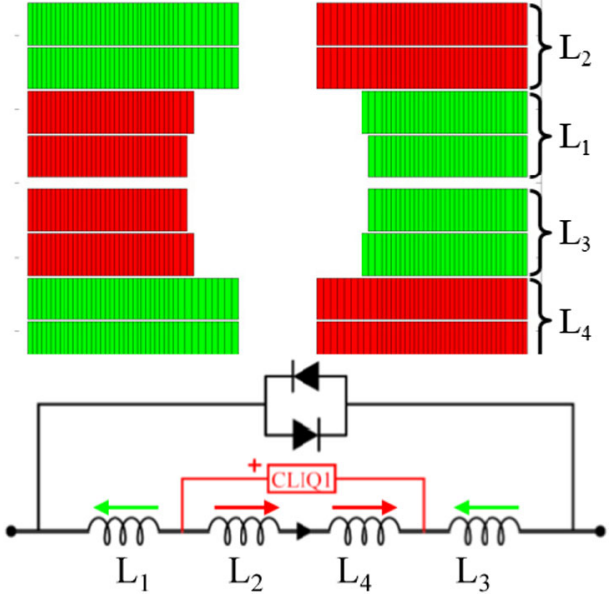

FIG. 17. Considered CLIQ connection scheme.

As shown in Fig. 17, the block design is composed of four layers per pole each made up of two double pancake coils. In this case, the easiest CLIQ connection scheme is obtained by fixing CLIQ leads to the joints between the double pancake coils. The red and green colors in Fig. 17 indicate the polarity of the CLIQ current in the cross section. Most of the coupling losses will be deposited in the cables close to the interface between positive and negative CLIQ currents, due to the high field derivative.

\section{B. CLIQ simulation}

When the CLIQ technology was originally introduced, none of the existing quench simulation tools were able to simulate a CLIQ discharge. For this reason, a new tool called TALES was developed in MathWorks Simulink by modeling the coil by a series of lumped elements [11]. This tool allows for the simulation of CLIQ alternating currents, interfilament and interstrand coupling losses, and includes the turn-to-turn transversal heat propagation.

Figure 18 shows the CLIQ currents obtained for the block design when the CLIQ unit is composed by a $20 \mathrm{mF}$

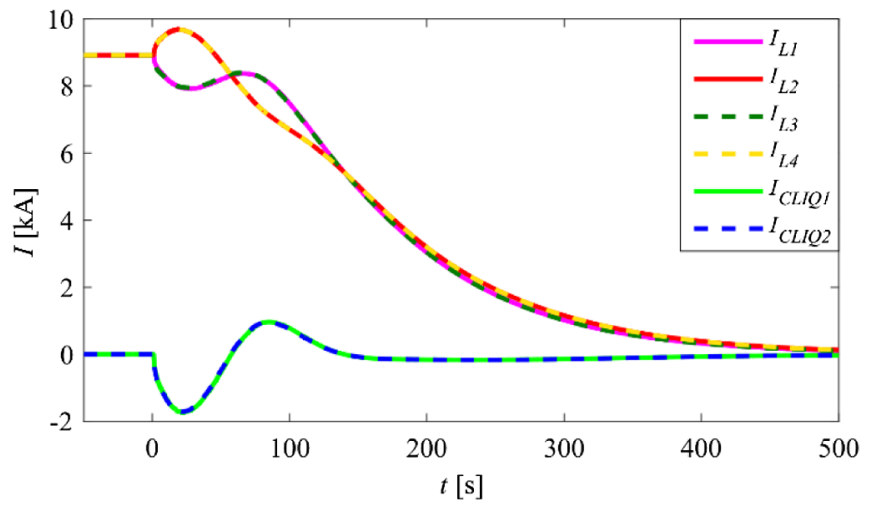

FIG. 18. Currents in different coil sections after a CLIQ discharge. The coils sections are described in Fig. 17. 
capacitor bank and $2 \mathrm{kV}$ initial voltage. The peak CLIQ current is $1.7 \mathrm{kA}$ and produces a rapid increase (decrease) in currents $I_{L 2}$ and $I_{L 4}\left(I_{L 1}\right.$ and $\left.I_{L 3}\right)$.

\section{CLIQ results}

Figure 19 shows the final temperature after a quench for the block design protected by a CLIQ system. The peak temperature is below $350 \mathrm{~K}$ while the temperature difference between low-field and high-field cables is of the order of $110 \mathrm{~K}$. As already explained, this is an intrinsic feature of the considered graded design and will lead to mechanical investigation.

Figure 20 shows the distribution of the voltage to ground when it reaches its peak value $(1.3 \mathrm{kV}$ at $t=140 \mathrm{~ms})$. The maximum interlayer voltage is $1.8 \mathrm{kV}$. The voltage distribution does not take into account the nonlinear distribution of the inductive voltage in the turns due to their different inductances. Nevertheless, also with this protection technology, it is confirmed that voltage to ground is a major issue in high-field, high-energy superconducting magnets and more efforts are needed, both in the magnet design and in the protection system design, to limit its value below $1 \mathrm{kV}$.

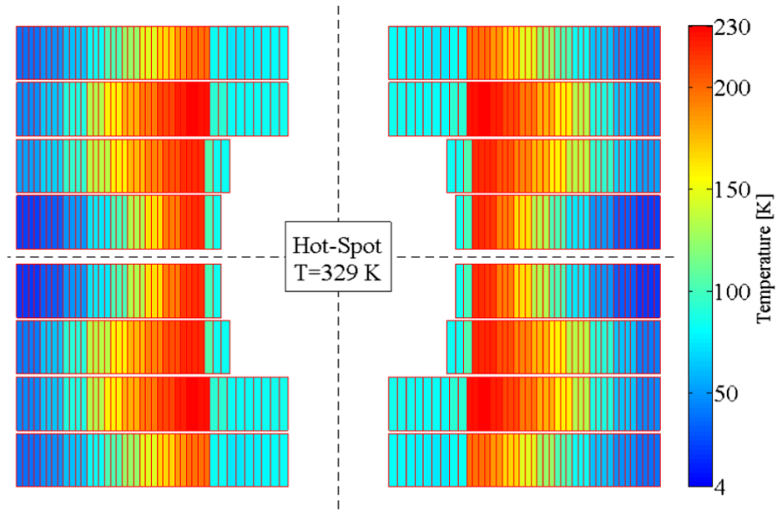

FIG. 19. Final temperature $(t=500 \mathrm{~ms})$ with CLIQ protection system.

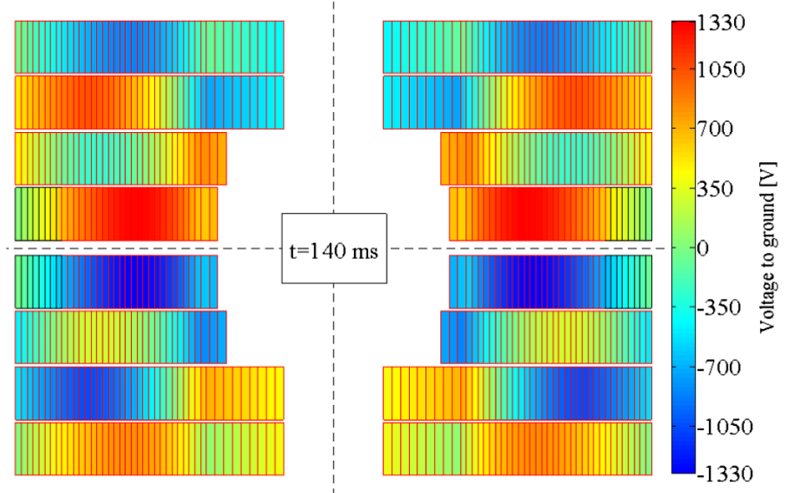

FIG. 20. Voltage-to-ground ( $t=140 \mathrm{~ms})$ with CLIQ protection system.

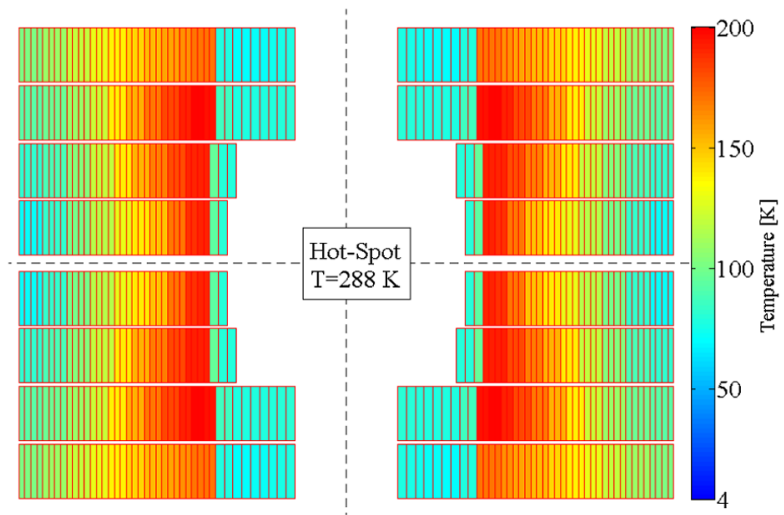

FIG. 21. Final temperature $(t=500 \mathrm{~ms})$ with CLIQ and quench heater protection systems.

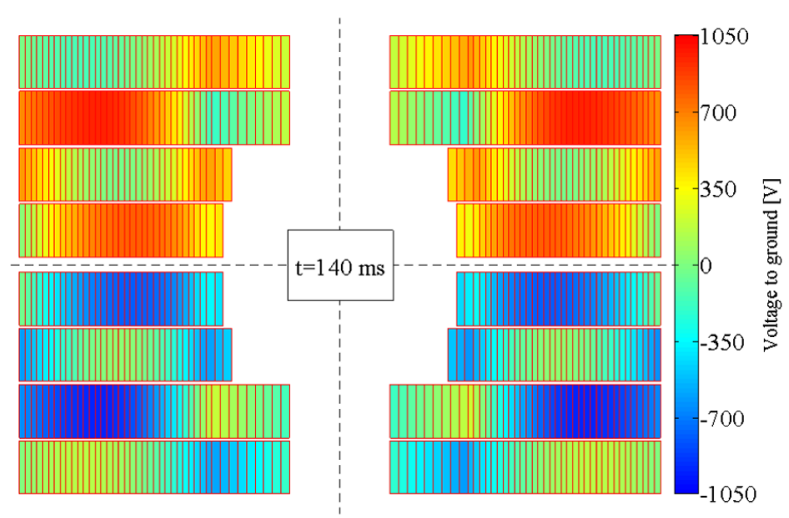

FIG. 22. Voltage to ground $(t=140 \mathrm{~ms})$ with CLIQ and quench heater protection system.

\section{CLIQ and quench heater results}

Figure 21 shows the final temperature after a quench in the block design when CLIQ and quench heaters are simultaneously present. Quench heaters are simulated in TALES following the uniform protection delay approach. The hot spot temperature is reduced to $290 \mathrm{~K}$, but the temperature difference between low-field and high-field cables is still around $100 \mathrm{~K}$.

Figure 22 shows the distribution of the voltage to ground when it reaches its peak value $(1.05 \mathrm{kV}$ at $t=140 \mathrm{~ms})$ for this second protection scenario. The peak voltage to ground is closer to its design constraint of $1 \mathrm{kV}$ and the maximum interlayer voltage is reduced to $1.15 \mathrm{kV}$.

\section{E. Comparison with quench heaters}

According to the comparison shown in Table V, even considering the simple CLIQ configuration of Fig. 17, CLIQ performances are similar to the ones of quench heaters. In order to improve the protection performance, it is foreseen that both protection technologies have to be included. 
TABLE V. Comparison of quench heaters and CLIQ performances.

\begin{tabular}{lccrc}
\hline \hline & $\begin{array}{c}T_{\max } \\
(\mathrm{K})\end{array}$ & $\begin{array}{c}\left|V_{\text {gnd }}\right| \\
(\mathrm{kV})\end{array}$ & $\begin{array}{c}V_{\text {turn-to-turn }} \\
(\mathrm{V})\end{array}$ & $\begin{array}{c}V_{\text {layer-to-layer }} \\
(\mathrm{kV})\end{array}$ \\
\hline QH (uniform delay) & 310 & 1.2 & 80 & 1.1 \\
QH (distributed delays) & 300 & 1.6 & 110 & 1.6 \\
CLIQ & 330 & 1.3 & 70 & 1.8 \\
CLIQ + QH (uniform & 290 & 1.05 & 34 & 1.15 \\
$\quad$ quench delay) & & & & \\
\hline \hline
\end{tabular}

\section{SUMMARY AND DISCUSSION}

In order to design compact, protectable, $16 \mathrm{~T}$ accelerator dipole magnets, the requirements for quench protection have been integrated into the design process from the beginning. In particular, assumptions were made of the maximum allowed hot spot temperature and voltages, and of the efficiency of a potential protection system. The considered magnet options were then analyzed, considering a sudden quench at $105 \%$ of nominal operation current, and a protection system with the projected efficiency. The resulting temperatures and voltages were compared to the maximum allowed values, namely $350 \mathrm{~K}$ of the peak temperature, and $1 \mathrm{kV}$ for the maximum voltage to ground during a quench.

The protection system has to be based on internal absorption of stored energy. This is obtained by transferring the entire winding to normal state within a certain quench delay time. In order to provide fast feedback during the magnet design, a single number was used to define the needed time margin, i.e., maximum quench delay time, for protection. The obtainable quench delay time was assumed to be $40 \mathrm{~ms}$, including $20 \mathrm{~ms}$ for detection and $20 \mathrm{~ms}$ for protection system. Following analysis either with heaters or CLIQ then confirmed that this is a reasonable choice.

Two new quench analysis tools were used for providing fast feedback. A Microsoft Excel spreadsheet was used by the magnet designers during the analysis to ensure sufficient amount of copper to satisfy the temperature requirements. Indeed, all the magnets had peak temperatures within the approved limits.

A more detailed code, Coodi, was then used for the analysis of temperature distributions and voltages for different preset heater delay distributions. Two cases were considered: A uniform 40 ms quench delay throughout the coil, and protection heaters which covered all coil turns. The computation suggested that the voltages were above the set limit of $1 \mathrm{kV}$ even in the nominal cases. It seems that this is due to the large inductive and resistive voltages and their nonuniform distribution that follow from the imposed design criterion of the cable dimensions. Therefore, this study suggests that solutions for FCC dipole magnets must have larger cables (i.e. higher current resulting in less turns and lower inductance) than the current state-of-the-art design options have.

Both the heater-based adiabatic quench simulations and CLIQ simulations with transverse (turn-to-turn) heat diffusion predict more than $100 \mathrm{~K}$ temperature gradient between the low-field and high-field cables of the coils. This needs to be further investigated. Lowering the $\mathrm{Cu} / \mathrm{non}-\mathrm{Cu}$ ratio in the high-field cable helps in reducing these temperature gradients.

Analysis of the CLIQ implementation in the block design was done using the TALES software, showing that the peak temperature could remain below $350 \mathrm{~K}$ with a CLIQ protection system. However, also this analysis showed that the voltages are too large.

This paper discussed the quench analysis which was performed to ensure that the magnets are not impossible to protect, and guide the direction of the next iteration in the design. The next step in the quench protection analysis is to sketch the protection scheme considering both heaters and CLIQ and the technical implementation of both [23].

\section{ACKNOWLEDGMENTS}

This work was supported by the following projects: The European Circular Energy-Frontier Collider Study, which receives funding from the European Union's H2020 Framework Programme under Grant Agreement No. 654305, and Advanced Numerical Computation Methods for Massive Parabolic Problems (Academy of Finland No. 287027).

[1] Future Circular Collider study website, available at https:// fcc.web.cern.ch/.

[2] EuroCirCol website, available at https://fcc.web.cern.ch/ eurocircol/.

[3] M. Benedikt, D. Schulte, and F. Zimmermann, Optimizing integrated luminosity of future hadron colliders, Phys. Rev. ST Accel. Beams 18, 101002 (2015).

[4] D. Schoerling, M. Durante, C. Lorin, T. Martinez, and F. Toral, Magnet cost model and targets, FCC week 2016, Rome, 2016.

[5] D. Tommasini et al., The $16 \mathrm{~T}$ dipole development program for FCC, IEEE Trans. Appl. Supercond. 27, 4000405 (2017).

[6] G. Ambrosio, $\mathrm{Nb}_{3} \mathrm{Sn}$ high field magnets for the high luminosity LHC upgrade project, IEEE Trans. Appl. Supercond. 27, 4002107 (2015).

[7] L. Imbasciati, Ph.D. thesis, Vienna University of Technology, Institute for Theoretical Physics, 2003.

[8] G. Ambrosio, Maximum allowable temperature during quench in $\mathrm{Nb}_{3} \mathrm{Sn}$ accelerator magnets, in Proceedings of WAMSDO: Workshop on Accelerator Magnet Superconductors, Design and Optimization, edited by E. Todesco (CERN, Geneva, 2013), pp. 43-46.

[9] H. ten Kate, (Multi)normal zone propagation velocity in high current density high field magnets, WAMSDO 2013, CERN, 2013. 
[10] E. Todesco, Quench limits in the next generation of magnets, in Proceedings of WAMSDO: Workshop on Accelerator Magnet Superconductors, Design and Optimization, edited by E. Todesco (Ref. [8]), pp. 10-16.

[11] E. Ravaioli, CLIQ: A new quench protection technology for superconducting magnets, Ph.D. thesis, University Twente and CERN, 2015.

[12] H. Felice et al., Instrumentation and quench protection for LARP $\mathrm{Nb}_{3}$ Sn magnets, IEEE Trans. Appl. Supercond. 19, 2458 (2009).

[13] E. Ravaioli et al., Advanced quench protection for the $\mathrm{Nb}_{3} \mathrm{Sn}$ quadrupoles for the high luminosity LHC, IEEE Trans. Appl. Supercond. 26, 4002006 (2016).

[14] T. Salmi et al., A novel computer code for modeling quench protection heaters in high-field $\mathrm{Nb}_{3} \mathrm{Sn}$ accelerator magnets, IEEE Trans. Appl. Supercond. 24, 4701810 (2014).

[15] T. Salmi, G. Chlachidze, M. Marchevsky, H. Bajas, H. Felice, and A. Stenvall, Analysis of uncertainties in protection heater delay time measurements and simulations in $\mathrm{Nb}_{3} \mathrm{Sn}$ high-field accelerator magnets, IEEE Trans. Appl. Supercond. 25, 4702305 (2015).

[16] T. Salmi and A. Stenvall, The impact of protection heater delays distribution on the hot spot temperature in a highfield accelerator magnet, IEEE Trans. Appl. Supercond. 26, 4001405 (2016).
[17] S. Russenschuck, ROXIE: A computer code for the integrated design of accelerator magnets, Proceedings, 6th European Particle Accelerator Conference, EPAC'98, Stockholm, Sweden, 1998, Vol. 1, pp. 2017-2019.

[18] E. Marquardt, J. Le, and R. Radebaugh, Cryogenic material properties database, in 11th International Cryocooler Conference, Keystone, 2000, edited by R. Ross, (Springer, Boston, MA, 2002), Vol. 11, pp. 681-687.

[19] J. Ruuskanen, A. Stenvall, V. Lahtinen, and E. Pardo, Electromagnetic nonlinearities in a Roebel-cable-based accelerator magnet prototype: Variational approach, Supercond. Sci. Technol. 30, 024008 (2017).

[20] V. Marinozzi, G. Bellomo, P. Fabbricatore, S. Farinon, M. Sorbi, and G. Volpini, Preliminary design of the EuroCirCol 16T $\cos \theta$ dipole for the Future Circular Collider, IEEE Trans. Appl. Supercond. 27, 4001205 (2017).

[21] C. Lorin, M. Durante, and M. Segreti, Status of 16 T dipole block design development, FCC week 2016, Rome, 2016.

[22] F. Toral, T. Martinez, and J. Munilla, EuroCirCol: 16T dipole based on common coils, FCC week 2016, Rome, 2016.

[23] T. Salmi, M. Prioli, A. Stenvall, A. P. Verweij, B. Auchmann, and V. Marinozzi, Suitability of different quench protection methods for the $16 \mathrm{~T} \mathrm{Nb}_{3} \mathrm{Sn}$ accelerator dipoles designed for the Future Circular Collider, IEEE Trans. Appl. Supercond. 27, 4702305 (2017). 